\title{
Modelagem Matemática e BIG DATA no desenvolvimento da literacia digital
}

Rose Grochot Gayeski

Rodrigo Dalla Vecchia

Marcus Vinicius Maltempi

\section{Resumo}

A sociedade está em crescente contato com as tecnologias digitais e a todo momento ações de indivíduos dão origem a vasta quantidade de dados. A reunião e cruzamento desses dados para gerar novas soluções e interpretações de problemas é chamada de Big Data. Consideramos que associado aos processos inerentes ao Big Data está um dos cernes de nossa pesquisa, a Literacia Digital, que está associada à interpretação e reflexão do uso de recursos e de informações provenientes das Tecnologias Digitais. Nosso objetivo principal é apresentar e analisar as potencialidades da Modelagem Matemática e do Big Data no desenvolvimento da Literacia Digital, norteados pela seguinte pergunta: Quais as potencialidades da Modelagem Matemática e do Big Data no desenvolvimento de habilidades relacionadas a Literacia Digital? O trabalho foi conduzido numa perspectiva metodológica qualitativa e envolveu tarefas usando recursos do Google Trends e Google Correlate. Os participantes da pesquisa foram estudantes do primeiro ano do ensino médio de uma escola localizada no interior do estado do Rio Grande do Sul. A análise dos dados destacou indícios da presença das habilidades Multitarefa, Navegação Transmídia e Simulação, as quais podem ter levado os estudantes ao desenvolvimento da Literacia Digital.

Palavras-chave: Modelagem Matemática. Big Data. Educação Matemática. 


\title{
Mathematical Modeling and BIG DATA in the development of digital literacy
}

\author{
Rose Grochot Gayeski \\ Rodrigo Dalla Vecchia \\ Marcus Vinicius Maltempi
}

\begin{abstract}
The Society is in growing contact with digital technologies and at all times the actions of individuals give rise to a vast amount of data. The gathering and crossing of this data to generate new solutions and interpretations of problems is called Big Data. We believe that associated with the processes inherent to Big Data is one of the cores of our research, Digital Literacy, which is associated with the interpretation and reflection of the use of resources and information from Digital Technologies. Our main objective is to present and analyze the potential of Mathematical Modeling and Big Data in the development of Digital Literacy, guided by the following question: What are the potentials of Mathematical Modeling and Big Data in the development of skills related to Digital Literacy? The paper was conducted in a qualitative methodological perspective and involved tasks using resources from Google Trends and Google Correlate. The research participants were first-year high school students in a school located in the interior of Rio Grande do Sul state. Data analysis highlighted evidence of the presence of multitasking abilities, Transmedia Navigation and Simulation, which may have led the students to the development of Digital Literacy.
\end{abstract}

Keywords: Mathematical Modeling. Big data. Mathematical Education 


\section{Introdução}

Neste artigo buscamos evidenciar a Modelagem Matemática (MM) como um meio para o desenvolvimento da Literacia Digital. Para tanto, além do entrelaçamento teórico que sustenta a análise, foram desenvolvidas tarefas usando o Google Correlate e o Google Trend, que são recursos de Big Data. Estas tarefas foram aplicadas a discentes do primeiro ano do Ensino Médio.

A problematização da pesquisa está ancorada nas transformações de mundo pelas tecnologias, em especial, pela Internet. Conforme Pessoa e Jamil (2015) o tratamento de dados provenientes da Internet está condicionando mudanças em áreas como: Educação, Saúde, Marketing, Administração, Logística, entre outras. Mudanças que tendem a se intensificar com o advento do protocolo IPv632, havendo um aumento de dados provenientes de dispositivos conectados à Internet - chamado de Internet das Coisas (IoT). Nesse contexto, os desafios que surgirem, segundo Pessoa e Jamil (2015), serão os relacionados à transformação desses dados em "informações úteis".

Esse volume de dados e seu tratamento, atualmente, são entendidos como Big Data (SANTOS, LEMES, 2014). Conforme IBM (2011) o Big Data está relacionado ao tratamento de bases de dados muito grandes e que, frequentemente, exigem técnicas e recursos diferentes dos que normalmente são utilizados. Nesse sentido, o "Big Data é mais do que simplesmente uma questão de tamanho, é uma oportunidade de encontrar insights em novos e emergentes tipos de dados e conteúdos, para tornar seu negócio mais ágil e para responder a perguntas que foram anteriormente consideradas fora de seu alcance” (IBM, s.d.).

O Big Data tem potencial de transformar, em um curto espaço de tempo, a realidade que conhecemos. Desse modo, entendemos que investigar aspectos relacionados às transformações proporcionadas por esse movimento torna-se fundamental à Educação, em especial, à Educação Matemática, ao passo que possibilita mudanças em sala de aula, nos processos de ensino e aprendizagem.

De fato, se atentarmo-nos à relação entre Tecnologias Digitais e Educação Matemática à sala de aula, podemos ver um cenário positivo. Com enfoque específico à Modelagem citamos os autores: Diniz (2007), Araújo (2002), Borba, Malheiros, Zulatto (2007), Dalla Vecchia e Maltempi (2009; 2010; 2012); Dalla Vecchia (2012; 2015), Javaroni (2007), Sinclair e Jackiw (2010), Chao, Empson e Shechtman (2010), Kazak (2010), Hills (2010) e Campbell (2010) e Carvalho (2018).

Nesse cenário, é possível encontrar distintas tendências quanto à finalidade do uso da MM no que diz respeito aos seus objetivos. Há pesquisas que trazem maneiras ou modos de

${ }^{32}$ Com o Protocolo de Internet versão 6, abreviado por IPv6, será possível acomodar a demanda exponencial de usuários e dispositivos que acessam a rede.

ISSN 2526-2882

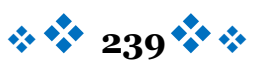


construir o conhecimento matemático, apresentando a MM, no âmbito da Educação Matemática, como método de ensino da Matemática. Nesse contexto a principal finalidade está no conteúdo a ser ensinado. Como exemplos de autores que tratam especificamente desse aspecto, destacamos Bassanezi (2002) e Biembengut (2007).

Há outros modos de compreender a MM que trazem finalidades distintas, como é o exemplo da visão sócio-crítica apresentada por Barbosa (2001). Nesta perspectiva, a Matemática não é vista como fim, mas como meio à proposição de argumentos críticos em relação a aspectos da realidade.

Nesses exemplos podemos identificar diferentes modos de conduzir a Modelagem no ensino. Assim, assumindo a Modelagem Matemática como um método de ensino de Matemática, questionamo-nos: Como a MM pode tangenciar as possibilidades e os desafios das tecnologias digitais, em particular, do Big Data? Que objetivos pedagógicos ou educacionais podemos ou queremos atingir quando realizamos esse entrelaçamento?

Nesse contexto vislumbramos a possibilidade de a MM ser o método para alcançar objetivos pedagógicos que extrapolam a própria Matemática e a colocam, dando sustentação às demais disciplinas do Programa Oficial do Ensino, em consonância as problemáticas da sociedade. Esse vislumbrar, nos leva a questão de pesquisa:

Quais as potencialidades da Modelagem Matemática e do Big Data no desenvolvimento de habilidades relacionadas a Literacia Digital?

Em busca de respostas, desenvolvemos a pesquisa, focados em tarefas envolvendo os recursos Google Trends e Google Correlate (detalhados na seção metodológica). Os participantes foram estudantes do primeiro ano do Ensino Médio, de uma escola no interior do estado do Rio Grande do Sul. Trata-se de análise qualitativa, com base na Literacia Digital defendida por Jenkins et al. (2009).

\section{A visão de modelagem matemática assumida}

Segundo Dalla Vecchia (2012, p. 123), a MM é “[...] um processo dinâmico e pedagógico de construção de modelos, sustentados por ideias matemáticas que se referem e visam encaminhar problemas de qualquer dimensão abrangida pela realidade”. Não se trata, portanto, de um processo necessariamente linear que segue etapas pré-determinadas e o modelo obtido é um exemplar da situação estudada que pode ser expresso por uma linguagem estruturada por ideias matemáticas. Para o autor, a MM é um processo educacional que extrapola a relação entre professor, aluno e o conteúdo, que se preocupa com o contexto que os envolve. 
Ao considerar essa visão entendemos ser possível observar a MM como um processo fluido e em constante transformação. Essa fluidez se deve, segundo o autor, a quatro aspectos relevantes: objetivo pedagógico, modelos/linguagem, problema e realidade, cujas características múltiplas de cada um se entrelaçam influenciando o processo, da mesma forma que as ondas formadas quando atiramos uma pedra em um lago de águas paradas, conforme Figura 1.

Figura 1 - A MM como um fluxo de quatro aspectos relevantes

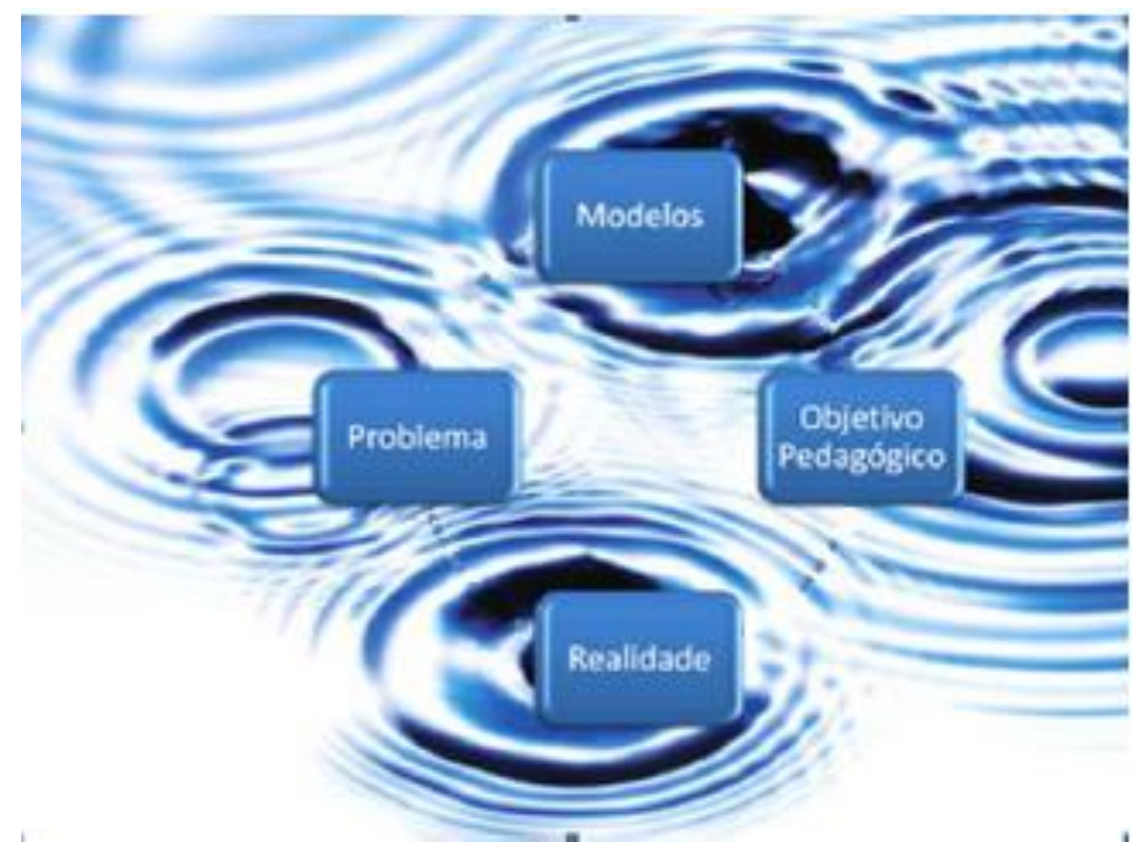

Fonte: Dalla Vecchia, 2012, p 127.

O objetivo pedagógico é “[...] o conjunto de fins ou metas que se deseja atingir quando se desenvolve qualquer tipo de proposta com os estudantes que visa a contribuir para o processo educacional" (DALLA VECCHIA, 2012, p. 71). Para tanto, o professor precisa dispor condições para que os estudantes efetivem suas ações de aprendizagem associadas ao processo de construção de um modelo matemático, o qual pode ser expresso por diferentes linguagens e perspectivas, baseado em ideias matemáticas, referindo-se à situação problema em estudo.

Na concepção de Dalla Vecchia (2012, p. 85), “[...] o modelo resultante do processo de MM será um, dentre a multiplicidade de aspectos que poderia assumir, sendo influenciado tanto pela linguagem que foi utilizada em sua construção, quanto pelas vivências e discussões dos envolvidos”. O problema é um conjunto de condições presentes na realidade do aluno e sua determinação influencia o processo de obtenção de uma solução, em que nem sempre o modelo encontrado será equivalente a solução do problema. Assim, o autor assume MM como 
um método que busca encaminhar problemas de qualquer dimensão da realidade em que a formulação dos modelos é sustentada por ideias matemáticas em um processo dinâmico.

Por último, o enfoque na realidade busca abarcar todas as dimensões da realidade, seja ela mundana ou cibernética. Desse modo, a referência a situações ocorridas no espaço cibernético também pode ser abarcada como referência para o modelo, havendo possíveis distinções qualitativas frente ao mundanamente vivenciado.

Diante desses quatro aspectos consideramos que essa perspectiva de MM vem ao encontro da proposta desenvolvida nos recursos do Big Data. O Google Correlate e o Google Trends disponibilizam inúmeras informações, que retratam os interesses de buscas das pessoas na Internet. O tratamento desse grande volume de informações obtido a partir de um modelo matemático retratado no recurso foi o grande desafio que os estudantes encontraram no decorrer do processo para atingir os objetivos pedagógicos, tais como, o desenvolvimento de habilidades da Literacia Digital.

\section{Literacia Digital}

Há vários autores que tratam da temática Literacia. Neste texto elegemos as definições de Martin (2006) e Jenkins et al (2009) para apresentar a concepção de Literacia Digital assumida para a análise do processo de intervenção.

Martin (2006) afirma que Literacia Digital abrange a consciência, a atitude e a capacidade dos indivíduos de utilizar recursos e facilidades digitais para identificar, acessar, gerenciar, integrar, avaliar, analisar e sintetizar recursos digitais, construir novos conhecimentos, criar expressões de mídia, e se comunicar com os outros. Essas capacidades são consideradas importantes pelo autor, pois os recursos digitais fazem parte do cotidiano das pessoas e é necessário saber utilizá-los de maneira consciente, sabendo identificar influência, impacto e potencialidade dos mesmos sobre a sociedade.

Jenkins et al (2009) afirmam que o desenvolvimento das habilidades de jogar, performance, simulação, apropriação, multitarefa, cognição distribuída, inteligência coletiva, julgamento, navegação transmídia, networking e negociação, levam à Literacia Digital.

Assim, Literacia Digital é a capacidade de ler e entender as diferentes informações expressas por meio de textos, imagens ou gráficos, nas mais diversas fontes do contexto digital para produzir/ampliar o conhecimento de maneira ética e com criticidade.

Embasados nessa literatura, nas próximas seções, apresentamos os aspectos metodológicos, as tarefas realizadas com os estudantes e seus respectivos resultados. De imediato podemos salientar o desenvolvimento de várias habilidades apontadas por Jenkins et al (2009), porém pelas limitações do texto, selecionamos três para exemplificar o 
desenvolvimento de habilidades que podem levar a Literacia Digital: Simulação, Multitarefa e Navegação Transmídia.

\section{Aspectos metodológicos e descrição das tarefas realizadas}

Na tentativa de obter respostas a pergunta: “Quais as potencialidades da Modelagem Matemática e do Big Data no desenvolvimento de habilidades relacionadas a Literacia Digital?” utilizamos uma metodologia qualitativa, uma vez que que a pesquisa qualitativa, segundo Bogdan e Biklen (1994) descreve os aspectos não mensuráveis do espaço educacional, impede a ação quantitativa, valoriza os aspectos descritivos e individuais e procura compreender o contexto, se importando com o processo e não só com os resultados.

A pesquisa se fundamentou, também, como qualitativa segundo Goldenberg (2004) e Bicudo (2011), pois envolveu um modelo qualitativo de descrição das situações que se constituíram no decorrer do processo, que buscou atingir aspectos do humano, sem passar pela mensuração, procurou olhar os aspectos reflexivos, não seguindo métodos previamente definidos.

Os recursos de Big Data utilizados na elaboração das tarefas, Google Trends e Correlate, identificam tendências e indicam relações entre dados aparentemente independentes. Segundo Santos e Lemes (2014) o Google Trends permite identificar a evolução de busca de termos específicos na internet, comparar a evolução e verificar o que as pessoas mais costumam pesquisar.

O Google Correlate, de acordo com Santos e Lemes (2014, p. 182), permite introduzir “[...] um termo de busca, uma série de dados temporais ou regionais e se obtém uma lista das consultas no Google cujas frequências seguem padrões que melhor se correlacionam com os dados [...]”. Podemos ver a tendência dos termos, o que possibilita, por exemplo, analisar tais tendências de mercado auxiliando na administração de empresas do ramo de vestuário e existem pesquisas cientificas na área da Economia, da Saúde, da Sociologia e Meteorologia.

Também, por meio do Google Correlate é possível associar um modelo construído com situações reais de busca na internet. Isso pode ser feito com o recurso "Search by Drawing", como mostra a Figura 2. Com este recurso é possível desenhar o gráfico de qualquer função e identificar a melhor correlação. Ressaltamos que os resultados obtidos podem ser diferentes um do outro, em função das particularidades de cada gráfico ao desenhar. 
Figura 2 - Resultado da busca no Google Correlate

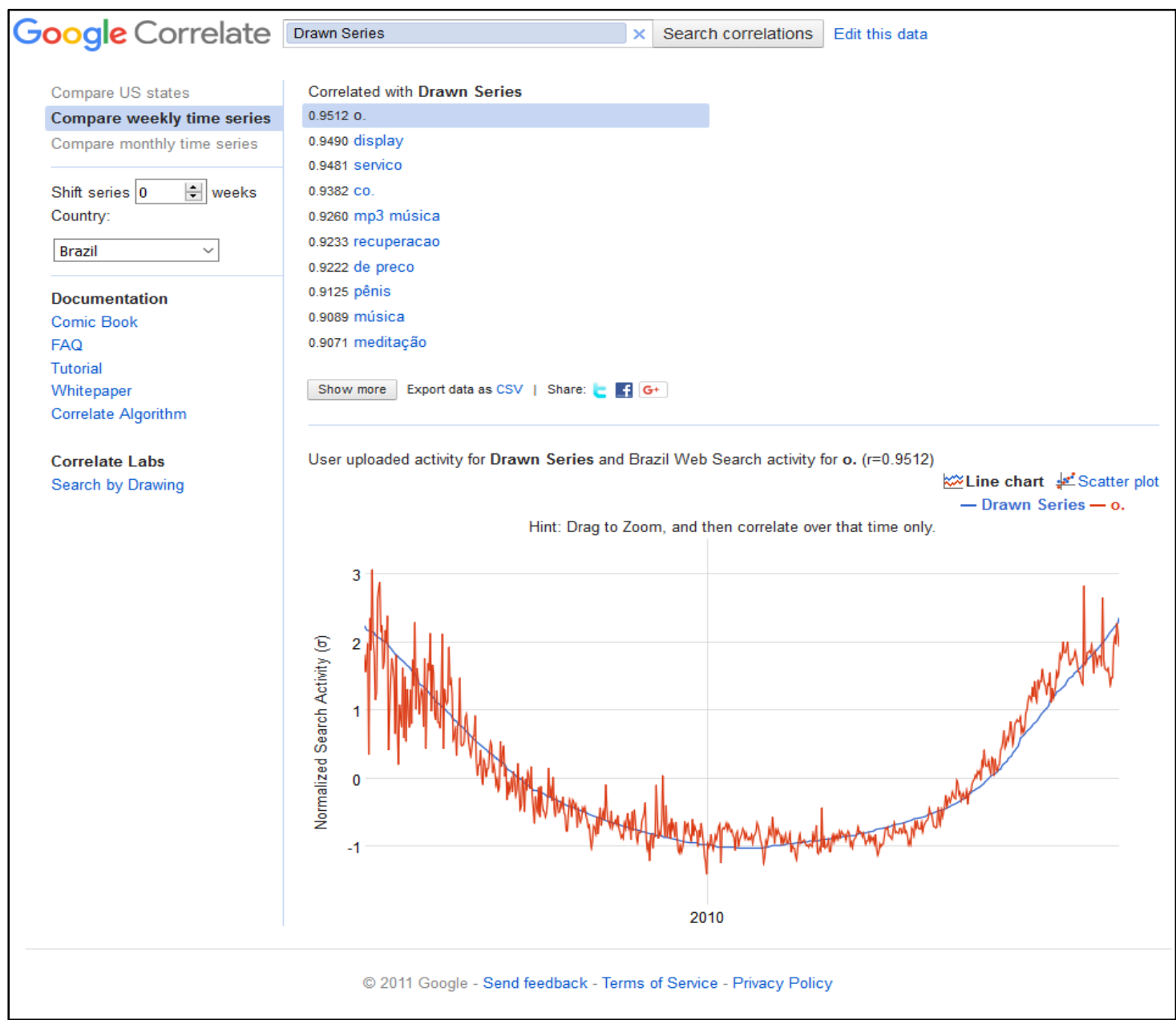

Fonte: site do Google Correlate

A produção de dados ocorreu com estudantes do primeiro ano do Ensino Médio de uma escola da rede privada de ensino localizada do município de Serafina Corrêa/RS. O desenvolvimento das tarefas se deu no Google Correlate e Google Trends, distribuídas em seis encontros, com duração de $1 \mathrm{~h} 40$ cada encontro. A turma em que o trabalho foi desenvolvido era composta por 11 estudantes. A escola conta com acesso a internet, porém não possui laboratório de informática, mas sempre que solicitado os estudantes levavam para a escola notebook ou smartphone. O registro dos dados produzidos durante o desenvolvimento das tarefas foi feito por meio do software Camtasia 33 e de anotações no caderno de campo.

No primeiro e segundo encontros os estudantes realizaram a exploração do Google Trends e do Google Correlate, respectivamente. O objetivo do terceiro encontro esteve relacionado à utilização do recurso Search by Drawing (pesquisar por desenho), para representar um gráfico que se referisse a uma função constante. No quarto e quinto encontros desenvolveu-se o processo de Modelagem Matemática por meio dos recursos explorados nos três primeiros encontros.

33 Camtasia Studio é uma marca registrada TechSmith, disponível em: http://www.techsmith.com/camtasia.html. Este software tem a finalidade de criar e editar vídeos, tanto capturados na tela do computador quanto gravações externas.

ISSN 2526-2882

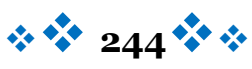


O processo de MM estava relacionado à exploração do conceito de Função Afim e a possibilidade de desenvolvimento de habilidades que podem levar a Literacia Digital. Foram iniciadas no quarto encontro, com a construção do gráfico da Função Afim, crescente ou decrescente, por meio do recurso Search by Drawing do Google Correlate seguido da observação dos termos que obedeciam ao comportamento do gráfico esboçado.

Salientamos que não foi previsto antecipadamente como seria realizada a discussão sobre o conceito de Função Afim, pois acreditava-se que a discussão surgiria naturalmente no momento em que a professora proporia a tarefa, uma vez que esse conceito não foi explicado em sala de aula pela professora.

Assim, o encontro iniciou com o desafio de desenhar um modelo matemático que se referisse a uma função de primeiro grau crescente ou decrescente no Google Correlate. De imediato, um membro de um dos grupos questionou: "Essa função, ao ser representada, vai ser uma reta?" Diante dessa pergunta, foi questionado aos demais estudantes sobre o que eles responderiam. Logo, houve respostas positivas e negativas, simultaneamente. Partindo das colocações feitas pelos estudantes, foi feita uma explanação e conversação com a participação deles sobre funções explicando que o gráfico de uma Função Afim é uma reta. Esse momento foi marcado por uma discussão participativa sobre a lei de formação da função de primeiro grau, crescimento e decrescimento, raiz da função, coeficiente angular e linear a partir das primeiras tentativas de construção no Google Correlate.

Após essa discussão, os estudantes foram orientados a escolherem um ou mais termos que chamaram a atenção e buscar informações sobre eles na internet, para entender a qual assunto o termo se referia, porque ele teve crescimento ou decrescimento nas pesquisas. A partir das informações colhidas, os estudantes foram convidados a produzir uma história real ou fictícia sobre o tema. Além disso, eles puderam explorar o recurso "Scatter plot" (gráfico de dispersão) para verificar como os pontos se aproximavam da função desenhada, como mostra a Figura 3.

Figura 3 - Gráfico de dispersão gerado pelo Google Correlate

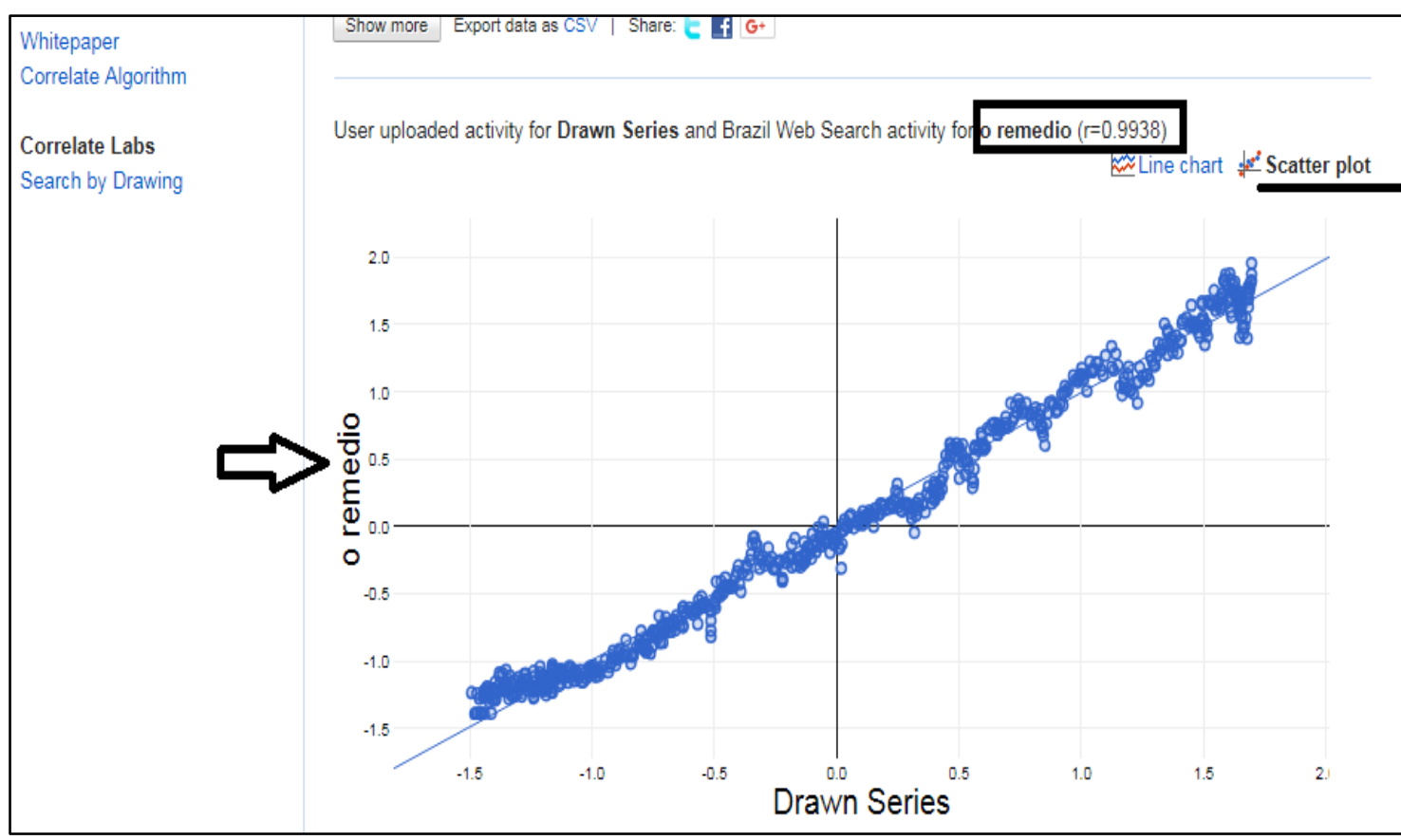

Fonte: a pesquisa ISSN 2526-2882 
Também, os estudantes exploraram o recurso zoom para selecionar alguns anos (Figura 4) e facilitar a construção da relação Matemática que se referia a função esboçada.

Figura 4 - Gráfico obtido com o recurso zoom do Google Correlate

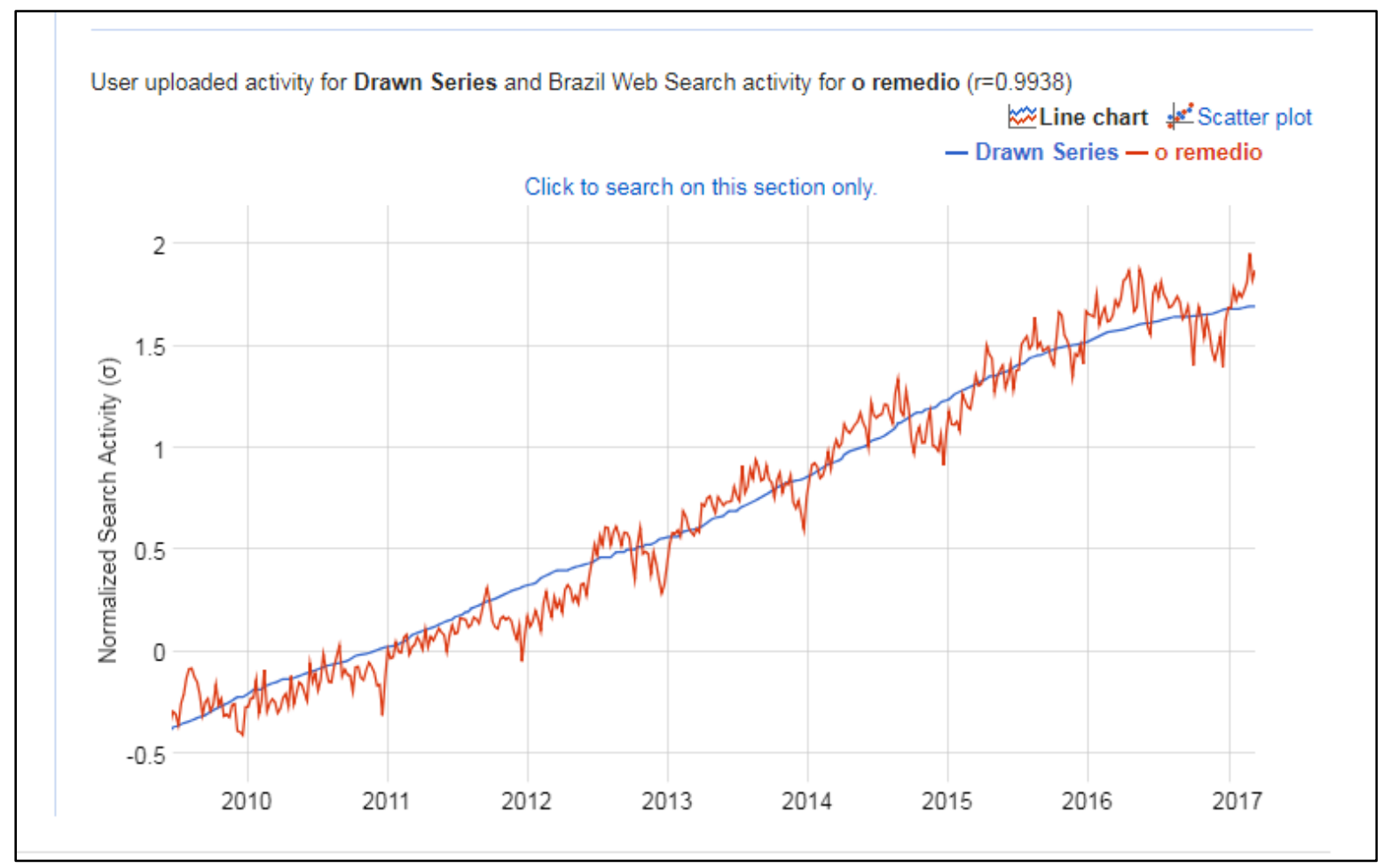

Fonte: a pesquisa

No prosseguimento das tarefas, os estudantes foram orientados a produzir um relatório, expressando a experiência com o recurso, justificando a escolha do termo, salvando os gráficos construídos e as respectivas palavras correlacionadas em um documento de texto. Ao final da aula os estudantes compartilharam os documentos e as gravações do software Camtasia na pasta do Google Drive para dar continuidade a tarefa que foi concluída e organizada, no quinto encontro, e apresentada no último encontro.

Cada grupo escolheu uma temática, a partir de suas construções, abordando quatro temas diferentes: o grupo 01 escolheu o termo "transferir", o qual se referia à transferência de pontos do cartão de crédito e relacionou com viagens; o Grupo 02 trabalhou com as temáticas, amizade, festa, gravidez na adolescência e valorização da vida a partir da escolha das palavras "amiga" e "demorando"; o tema do Grupo o3 foi medicamentos e doenças, sendo que as palavras escolhidas por eles foram "Omeprazol" e "Amlodipina"; e o Grupo o4 escolheu a palavra "cifras" e escreveu uma história de amor.

\section{Análise}

Para o recorte do presente artigo, apresentaremos os indícios de três das onze habilidades apontadas por Jenkins et al (2009), a saber, Simulação, Multitarefa e Navegação 
Transmídia. Entendemos que os estudantes vivenciaram um processo que se assemelha ao que Jenkins et al. (2009) chamam de Simulação. Esse processo inicia com a preocupação em esboçar no Google Correlate uma reta, durante a realização da tarefa, que pode ser verificada na frase da aluna A quando diz: “Como faz uma reta crescente?” Essa inquietação remete a uma rápida reflexão, apresentada pelo Aluno M, que diz: "É só desenhar crescendo". Então, a Aluna A tenta desenhar, mas logo afirma: "Só que olha, agora caiu” (Excerto 01). Ao mencionar isso ela se refere à imagem da Figura 5. Segue o Excerto 01 que mostra a situação mencionada.

\section{Excerto o1: Parte da conversa dos estudantes do Grupo 01 no quarto encontro}

Aluna A: Como faz uma reta crescente?

Aluno M: É só desenhar crescendo.

Aluna A: Desenhandooo.

Aluna A: Só que olha, agora caiu.

Aluno L: É só pegar aqui embaixo (mostra na tela).

Aluna A: Não. É só ignorar essa parte (decrescendo) e pronto. Dar zoom.

Aluna L: Não dá, eu acho. Vamos ver o que cresceu bastante e depois decaiu.

Verificamos que a Aluna A sugere: "É só ignorar essa parte e pronto”, porém a Aluna L menciona que não é possível ignorar. Ao solicitar à professora, e questioná-la se o gráfico podia ser dessa forma (Figura 5), recebem a resposta que era necessário refazê-lo, que dessa forma ele não se referia a um modelo de uma função de primeiro grau crescente.

Assim, partiram para sucessivos testes até conseguir traçar uma reta crescente de modo adequado. A habilidade se manifesta via tentativa e erro em esboçar o gráfico, de acordo com Sápiras, Dalla Vecchia e Maltempi (2015) a Simulação ocorre durante o processo de construção baseado em tentativas que podem gerar erros ou acertos.

Figura 5 - Gráfico esboçado pelo Grupo 01 no quarto encontro

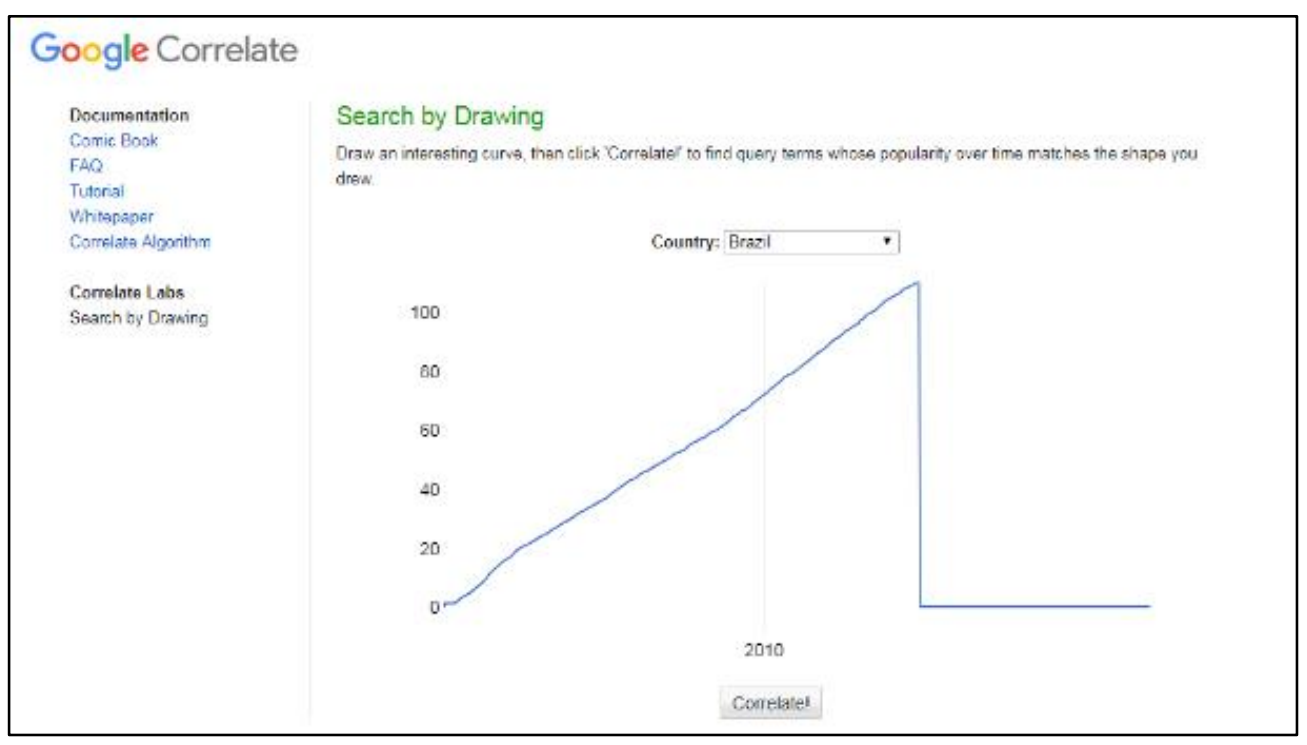

Fonte: a pesquisa

ISSN 2526-2882

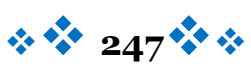


Após o esboço do gráfico de forma correta, os estudantes, do Grupo 01, selecionaram um termo que se referisse ao modelo desenhado, conforme a Figura 6 e buscaram informações na Internet, a partir do hiperlink da palavra escolhida por eles, no caso desse grupo eles escolheram a palavra "transferir" para investigar.

Figura 6 - Gráfico esboçado corretamente pelo Grupo 01 no quarto encontro

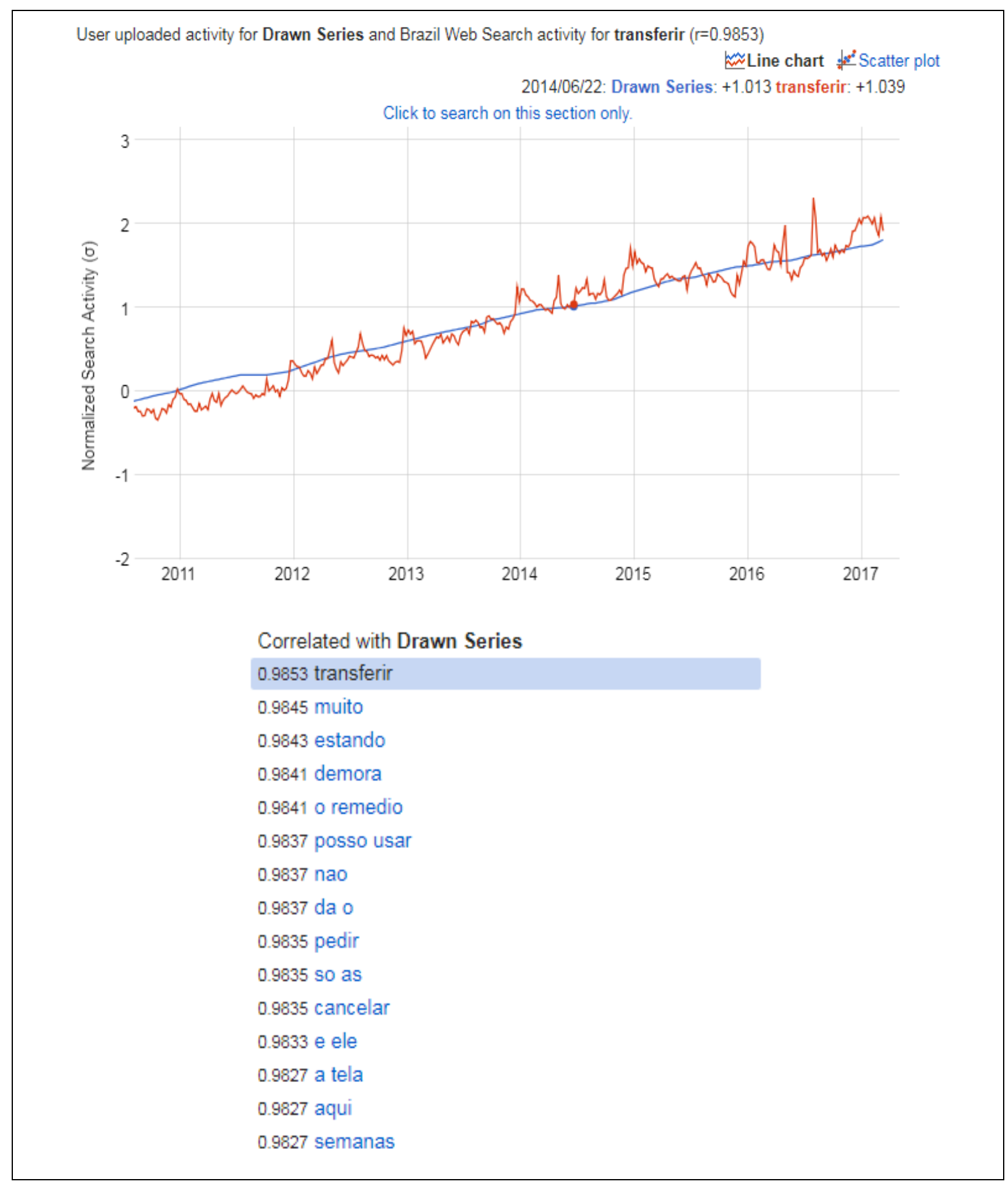

Fonte: a pesquisa

ISSN 2526-2882 
A pesquisa feita pelos estudantes mostrou que o termo "transferir" estava associado às promoções relacionadas a cartões de créditos (Figura 7), que permitem acumular "milhas" (pontos) que podem ser transferidas em descontos em viagens aéreas.

Figura 7 - Pesquisa realizada pelo Grupo 01 na Internet

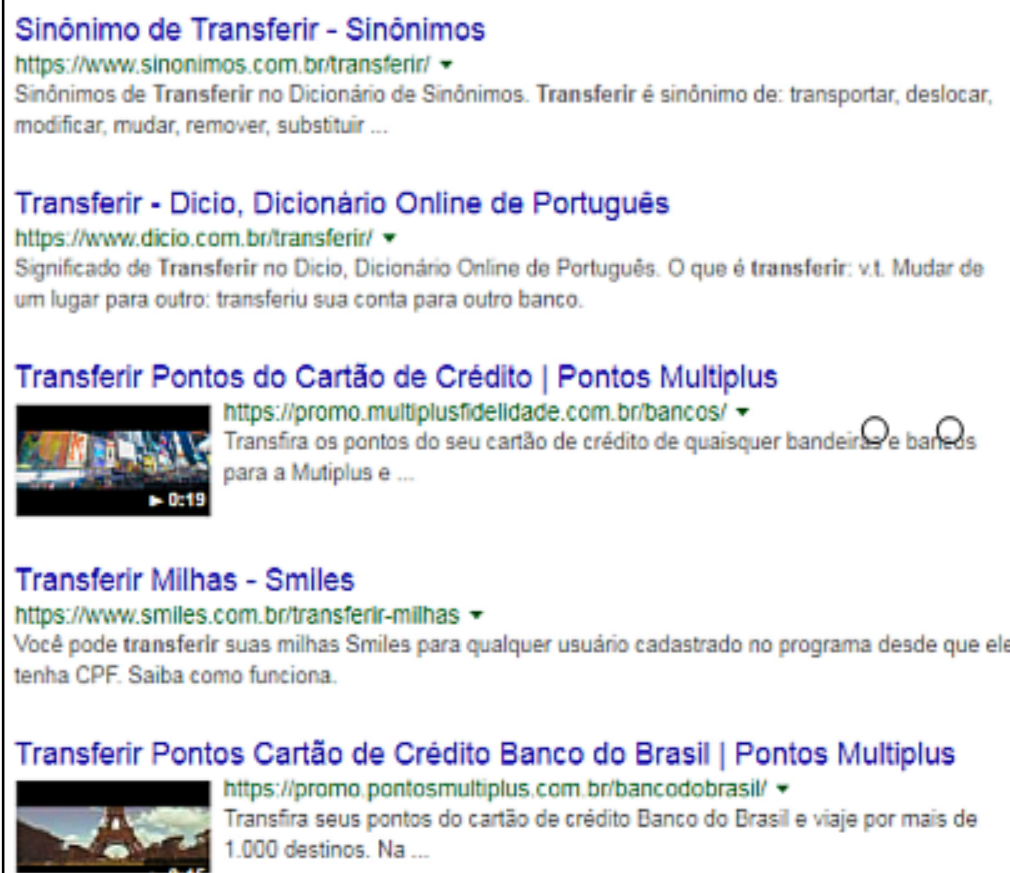

Fonte: a pesquisa

Os estudantes, também, buscaram informações no Google Trends sobre "cartão de crédito" e "milhas" para verificar como era a busca por esses termos nos últimos 12 meses na base de dados do Brasil. O gráfico gerado pelo recurso (Figura 8) apresentou uma relação de crescimento nas pesquisas.

Figura 8 - Pesquisa realizada pelo Grupo 01 no Google Trends

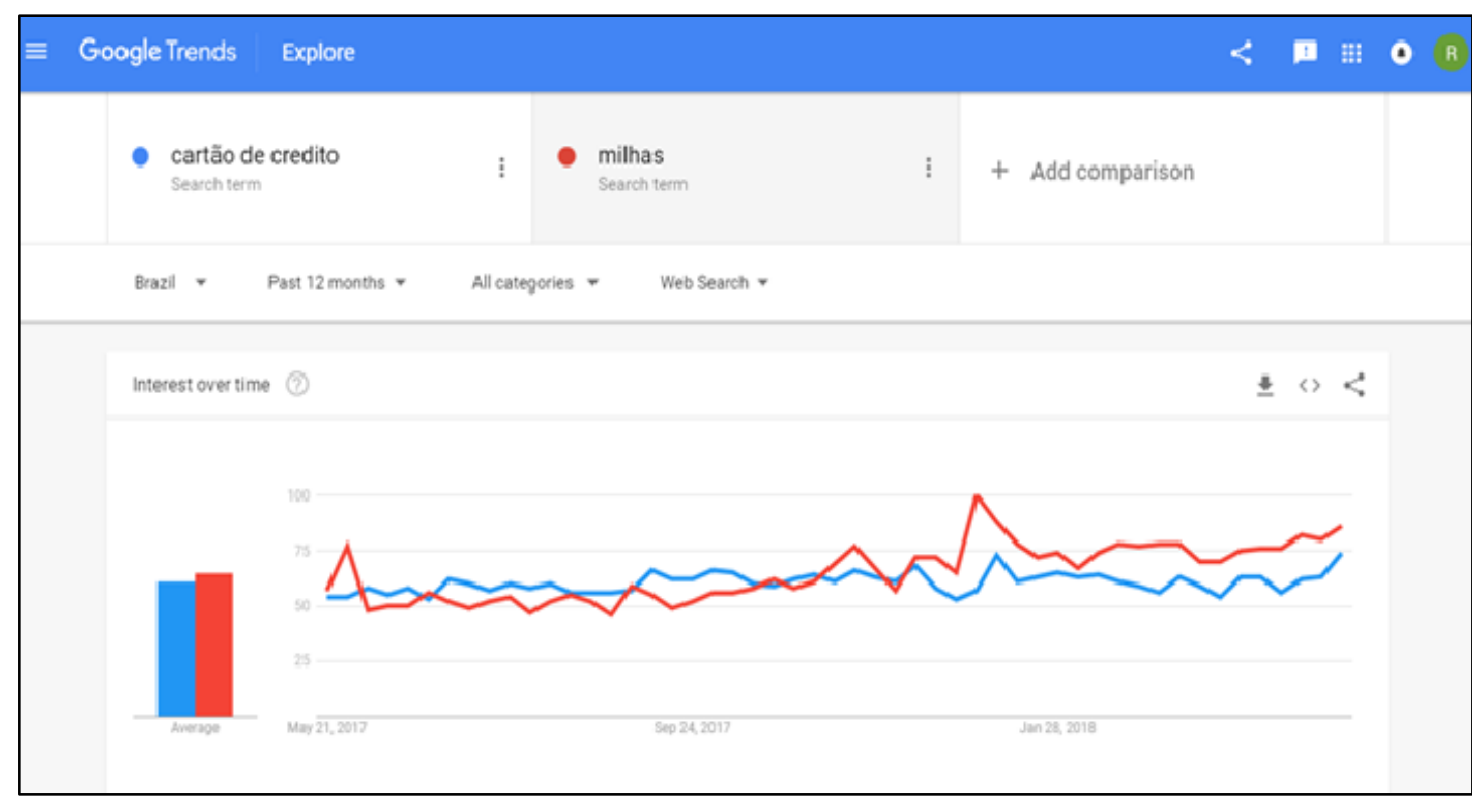

Fonte: a pesquisa

ISSN 2526-2882 
Os pontos de máximo, do termo "milhas", foram nos meses de janeiro (2018) e maio (2017). Esse aspecto foi observado pelos estudantes, uma vez que, ao buscar informações na Internet, descobriram que naqueles meses havia descontos em passagens aéreas, o que pode ter motivado mais pesquisas. O gráfico gerado pelo recurso (Figura 8) apresentou uma relação de crescimento nas pesquisas.

A partir da observação do gráfico eles perceberam detalhes importantes, como quando a aluna A diz que possui as mesmas datas do Google Correlate, interligando as informações adquiridas nos dois recursos, mostrando que é exatamente o que Jenkins et al (2009) entendem por Multitarefa. Podemos salientar que essa habilidade requer atenção para perceber as informações relevantes no campo de pesquisa e estabelecer a relação entre as informações que chegam de diferentes fontes.

Ao mesmo tempo foi observada a habilidade Navegação Transmídia, que para Jenkins et al. (2009) corresponde a capacidade de acompanhar o grande fluxo de informações recebidas por meio de múltiplas plataformas e de diferentes formas como imagens, vídeos, textos, áudios ou simulações. Podemos pensar de maneira semelhante sobre aquilo que queremos expor, compartilhar, postar ou comunicar, para selecionar a melhor técnica e o melhor recurso para fazer isso. Aos indivíduos que navegam, cabe analisar os inúmeros conteúdos disponíveis e fazer a escolha daquilo que é mais importante para o momento. Observamos isso no Excerto 02 do quarto encontro do Grupo 01, quando os estudantes visitam páginas da Internet relacionadas ao termo transferir, para incrementar a narrativa que estavam produzindo, com o objetivo de entender como funciona a transferência de pontos do cartão de crédito.

\section{Excerto 02: Parte da conversa do Grupo o1 no quarto encontro}

Aluna A: Pesquisem como transferir pontos do cartão de crédito. (...)

Aluna L: Aqui deve ter os termos e condições. (Referindo-se a Figura 9) (...)

Aluna L: Olha! Aqui tem como transferir. 
Figura 9 - Pesquisa realizada pelo Grupo 01 sobre como efetuar a transferência de pontos do cartão de crédito

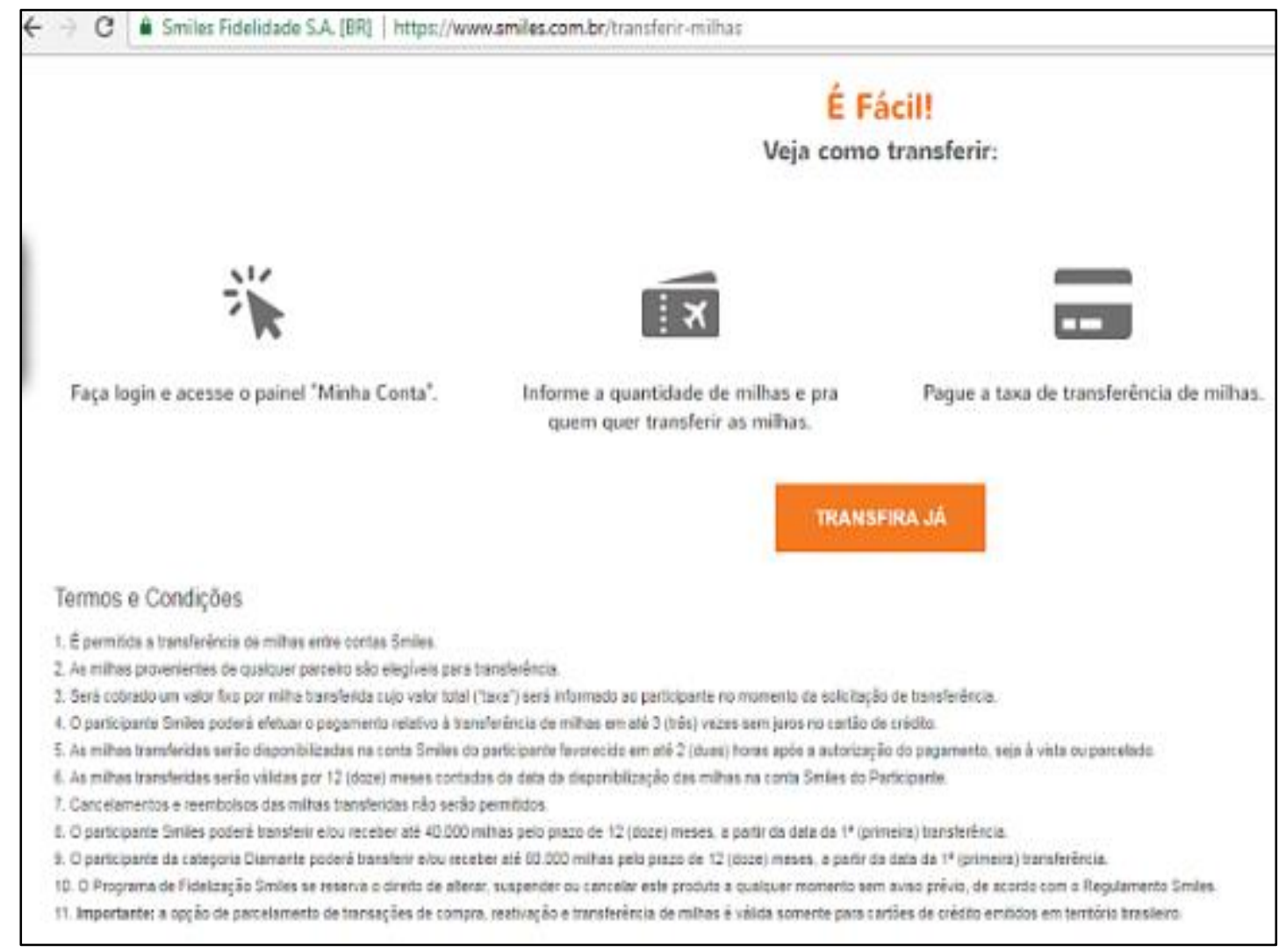

Fonte: a pesquisa

Os estudantes diante dos vários sites disponibilizados pela Internet em sua pesquisa selecionam o site da Figura 9, o qual foca nos termos e condições para efetuar a "transferência de pontos do cartão de crédito", mostrando necessidade de selecionar, com discernimento, as informações relevantes para o momento. Além disso, no instante em que o grupo estava construindo a história o Aluno M sugere: "Olha! Achei esse vídeo sobre Paris, acho que vai ajudar". Eles assistem ao vídeo34 que possivelmente, no nosso entendimento, serviu de inspiração para continuar a narrativa, tarefa que foi apresentada como produto final desse processo no último encontro. Então, de acordo com a definição de Navegação Transmídia de Jenkins et al. (2009) evidenciaram-se características dessa habilidade quando os estudantes transitaram por mídias escritas, por vídeos e imagens ao inserir hiperlinks na narrativa.

A habilidade também se fez presente nas tarefas desenvolvidas pelo Grupo 02 no quinto e no sexto encontros. Os estudantes produziram um vídeo que foi apresentado juntamente com a narrativa. Para produzir o vídeo os componentes do grupo navegaram por diferentes sites, redes sociais e galerias de seus smartphones para resgatar momentos vivenciados pela turma, atingindo a habilidade da Navegação Transmídia. Segundo Jenkins et al. (2009) essa habilidade corresponde a capacidade de seguir um fluxo de informações expostas em diferentes mídias, seja uma imagem, um vídeo, um áudio,

34 vídeo que os estudantes assistiram encontra-se no link: https://www.youtube.com/watch?v=hREcgljzKPA . 
um texto ou uma simulação, para interagir, selecionar e compartilhar as informações relevantes. O grupo demonstrou essa segurança de transitar por diferentes informações e selecionar aquelas que consideraram mais importantes tanto para produzir a narrativa como para produzir o vídeo. Esses aspectos podem ser exemplificados no momento que o Aluno B sugere usar o filme "Simplesmente Acontece” 35 como mais uma inspiração para produzir a narrativa.

Entendemos que os estudantes desenvolveram a habilidade da Navegação Transmídia, pois de acordo Jenkins et al. (2009) transitaram por múltiplas plataformas, uma vez que o grupo assistiu ao vídeo do trailer do filme, além do que transitou pelas mídias escritas acessando Wikis (https://pt.wikipedia.org/wiki/Love,_Rosie) do filme "Simplesmente Acontece" (Figura 10). De acordo com o autor, é possível navegar nas diferentes mídias para extrair aquilo que nos interessa para produzir algo novo, que pode ser exposto de formas diferentes daquelas acessadas e foi o que o grupo fez, criando a narrativa: "Para cada ação, uma reação".

Figura 10 - Site acessado pelo Grupo 02 durante a construção da narrativa

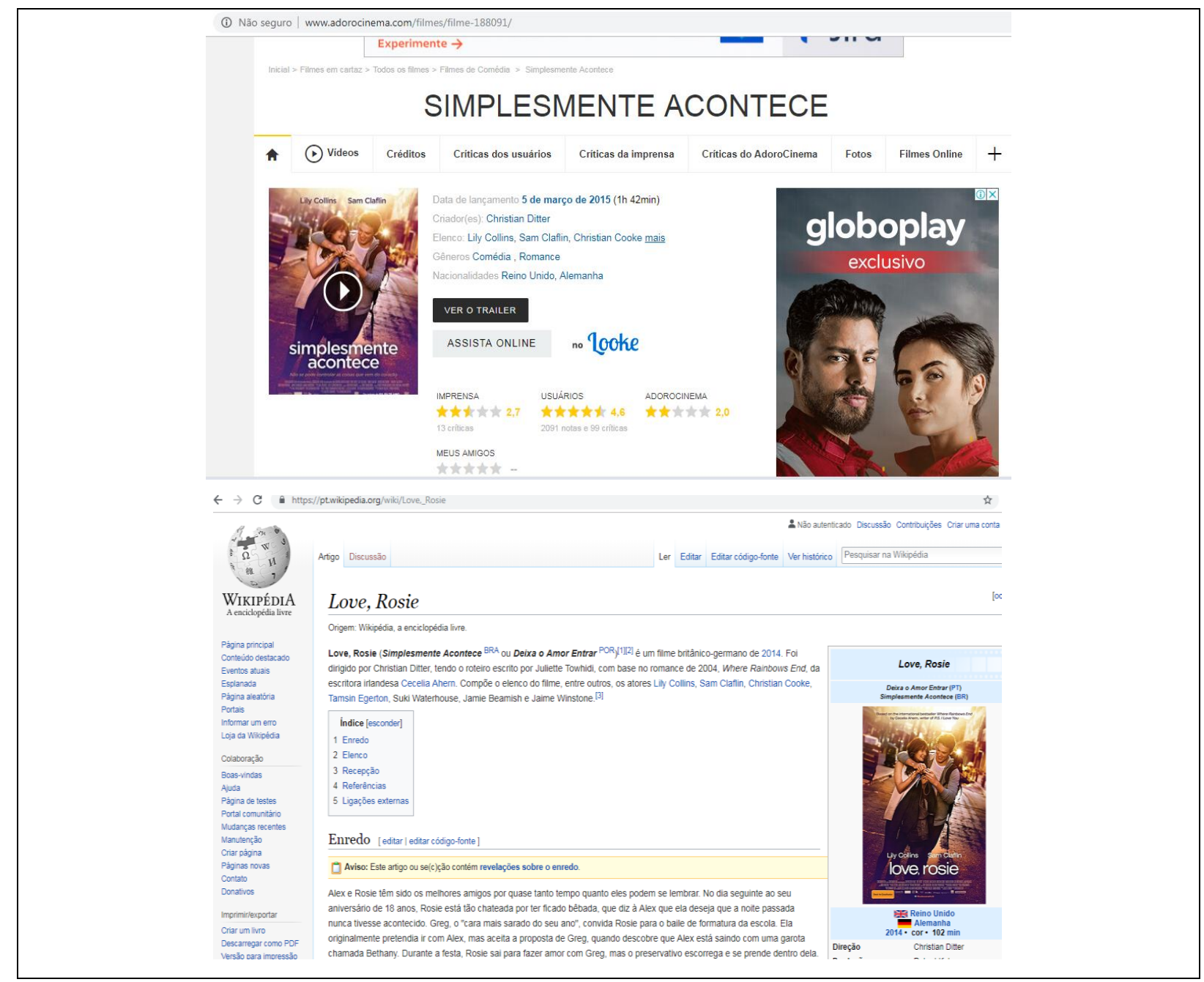

Fonte: a pesquisa

$35 \mathrm{O}$ filme trata de uma história de dois jovens que experimentaram juntos as dificuldades amorosas, familiares e escolares. Pode se https://www.youtube.com/watch?v=LEO2sN6MmHo . 
Podemos observar que os alunos desenvolveram a habilidade de Navegação Transmídia, pois eles navegaram em diferentes plataformas e extraíram aquilo que era pertinente para a construção das narrativas, uma vez que a característica fundamental dessa habilidade, segundo Jenkins (2009, é acompanhar o grande fluxo de informações que eles encontram em múltiplas plataformas e selecionar aquilo que é importante para produzir algo novo.

\section{Considerações finais}

$\mathrm{Na}$ introdução deste artigo nos questionamos acerca dos objetivos pedagógicos relacionados a interseção entre MM e Tecnologias Digitais. Nesse sentido colocamos em suspensão a possibilidade de assumir a MM como um meio para construção da Literacia Digital e relatamos nossa investigação, buscando consolidar esse aspecto. Ao final deste processo consideramos, baseados nos dados apresentados e nas análises feitas, que a associação dos recursos do Big Data ao processo de MM mostrou a possibilidade do desenvolvimento de habilidades relacionadas à Literacia Digital. Em particular, destacamos as habilidades de Simulação, Navegação Transmídia e Multitarefa.

Observamos que o ambiente criado pelas tecnologias, no nosso caso, pelos recursos do Big Data, possui potencial próprio ao ser vinculado a educação. Entendemos que por meio de tarefas, como as descritas neste artigo, é possível desvelar importantes potencialidades dessa associação, mostrando um processo no qual o aluno é protagonista/construtor de seu próprio conhecimento, interagindo com as mídias e com os colegas, permitindo o desenvolvimento da criticidade dos estudantes, preparando-os para serem mais cautelosos com uso dos recursos tecnológicos, uma vez que eles diariamente costumam expor sua realidade e seus interesses nas diferentes plataformas digitais.

Como pesquisas futuras associadas a esta investigação, destacamos a possibilidade de considerar os dados produzidos pelos estudantes como narrativas. Neste ínterim, buscaremos compreender a relação entre os produtos do estudo e sua relação com a experiência vivida no ambiente dado pela realidade do mundo cibernético. Espera-se com ela produzir inquietações acerca do próprio processo de Modelagem Matemática, quando a realidade está proporcionada pelas tecnologias digitais.

\section{Referências Bibliográficas}

ARAÚJO, J. L. Cálculo, tecnologias e modelagem matemática: as discussões dos alunos. Rio Claro: UNESP, 2002. Tese (Doutorado em Educação Matemática), Instituto de Geociências e Ciências Exatas, Universidade Estadual Paulista, Rio Claro, 2002. 
BARBOSA, J. C. Modelagem na Educação Matemática: Contribuições para o Debate Teórico. In: Reunião Anual da ANPED, 24. 2001, Caxambu/RJ. Anais. Rio Janeiro: ANPED, 2001.

BARBOSA, J. C. Modelagem na Educação Matemática: Contribuições para o Debate Teórico. In: Reunião Anual da ANPED, 24. 2001, Caxambu/RJ. Anais. Rio Janeiro: ANPED, 2001.

BASSANEZI, R. C. Ensino-aprendizagem com Modelagem Matemática. Editora Contexto, 2002.

BICUDO, M. A. V. Pesquisa Qualitativa e Pesquisa Qualitativa: segundo a Abordagem Fenomenológica. São Paulo: Cortez, 2011

BIEMbengut, M. S.; HEIN, N. Modelagem Matemática no Ensino. São Paulo: Contexto, 2007.

BOGDAN, R.; BIKLEN, S. K. Investigação Qualitativa em Educação - Uma introdução à teoria e aos métodos. Coleção Ciências da Educação. Portugal: Porto, 1994.

BORBA, M.de C.; MALHEIROS, A. P. S.; ZULATTO, R. B. A. Educação a Distância online. Belo Horizonte: Autêntica, 2007.

CAMPBELL, S. R. Mathematical Modeling and Virtual Environments. In: LESH, Richard; GALBRAITH, Peter; HAINES, Christopher R.; HURFORD, Andrew. (Org.). Modeling Students' Mathematical Modeling Competences. New York: U.S.A., Springer, 2010.

CARVALHO, F. J. R. Introdução à programação de computadores por meio de uma tarefa de Modelagem Matemática na Educação Matemática. Dissertação de Mestrado em Ensino. Programa de Pós-Graduação Stricto Sensu em Ensino. Universidade Estadual do Oeste do Paraná. Foz do Iguaçu, 2018.

CHAO, T.; EMPSON, S. B.; SHECHTMAN, N. A principal Components Model of Simcalc Mathworlds. In: LESH, Richard.; GALBRAITH, Peter; HAINES, Christopher R.; HURFORD, Andrew. (Org.). Modeling Students Mathematical Modeling Competences. New York: U.S.A., Springer, 2010.

DALLA VECCHIA, R. A Modelagem Matemática e a realidade no mundo cibernético. Tese de Doutorado em Educação Matemática. Programa de Pós-Graduação do Instituto de Geociências e Ciências Exatas do Campus de Rio Claro. Universidade Estadual Paulista Júlio de Mesquita Filho. Rio Claro, 2012.

DALLA VECCHIA, R. The relationship between Big Data and Mathematical Modeling: a discussion in a Mathematical Education scenario. Themes in Science and Technology Education, v. 8, p. 23, 2015. 
DAlla VECCHIA, R.; MAlTEMPI, M. V. Tecnologias Digitais e Percepção da Realidade: Contribuições para a Modelagem Matemática. In: X Encontro Nacional de Educação Matemática. Anais... Bahia, 2010. p. 1- 10

DAlla VECCHIA, R.; MALTEMPI, M. V. Ensaio Sobre a Modelagem Matemática e o Virtual. In: XIII Encontro Brasileiro de Estudantes de Pós-Graduação em Educação Matemática, Anais... Goiânia, 2009. p. 1- 15

DALla VECCHIA, R.; MALTEMPI, M. V. Modelagem Matemática e Tecnologias de Informação e Comunicação: a realidade do mundo cibernético como um vetor de virtualização. Bolema. v. 26, $\mathrm{n}^{\circ}$ 43, p. 963 - 990. Rio Claro, 2012

DALLA VECCHIA, R.; MALTEMPI, M. V.; BORBA, M. de C. The Construction of Eletronic

Games as an Environment for Mathematics Education. In: Tom Lowrie; Robyn Jorgensen. (Org.). Digital Games and Mathematics Learning. 1ed. Heidelberg: Springer, v. 1, p. $55-69,2015$.

DINIZ, L. do N. O Papel das Tecnologias de Informação e Comunicação nos Projetos de Modelagem Matemática. 2007. Dissertação (Mestrado em Educação Matemática) - Instituto de geografia e Ciências Exatas, Universidade Estadual Paulista, Rio Claro, 2007.

GOLDENBERG, M. A arte de pesquisar: como fazer pesquisa qualitativa em Ciências Sociais. 8a. ed. Rio de Janeiro: Record, 2004.

HILLS, T. Investigating Mathematical Search Behavior Using Network Analysis. In: LESH, Richard; GALBRAITH, Peter; HAINES, Christopher R.; HURFORD, Andrew. (Org.). Modeling Students' Mathematical Modeling Competences. New York: U.S.A., Springer, 2010.

IBM. What is big data? s.d. Armonk, NY: IBM, 2011. Disponível em: <http://www01.ibm.com/software/data/bigdata/>. Acesso em: 04 jan. 2019.

JAVARONI, S. L. Abordagem geométrica: possibilidades para o ensino e aprendizagem de Introdução às Equações Diferenciais Ordinárias. UNESP, 2007. Tese (Doutorado em Educação Matemática), Instituto de Geociências e Ciências Exatas, Universidade Estadual Paulista, Rio Claro, 2007.

JENKINS, H.; CLINTON, K.; PURUSHOTMA, R.; ROBISON, A. J.; WEIGEL, M. Confronting the Challenges of Participatory Culture: Media Education for the 21 ${ }^{\text {st }}$ Century. The MacArthur Foudation, Chicago, 2009. Disponível em: $<$ https://www.curriculum.org/secretariat/files/Sept3oTLConfronting.pdf >. Acesso em: 02 jan, 2018.

KAZAK, S. Modeling Random Binomial Rabbit Hops. In: LESH, Richard; GALBRAITH, Peter; HAINES, Christopher R.; HURFORD, Andrew. (Org.). Modeling Students' Mathematical Modeling Competences. New York: U.S.A., Springer, 2010.

$$
\text { ISSN 2526-2882 }
$$


MARTIN, A. A European Framework for Digital Literacy. Nordic Journal of Digital Literacy, v. 2, n. 1, p. 151 - 161, 2006.

PESSOA, C. R. M.; JAMIL, G. L. A Internet Das Coisas: será a Internet do futuro ou está prestes a se tornar a realidade do presente? Disponível em <http://www.fumec.br/revistas/eol/article/download/2961/1732> Acesso em 13 de dezembro de 2015 .

SANTOS, R. P. dos.; LEMES, I. L. Aprender-com-Big-Data no Ensino de Ciências. Acta Scientia, v.16, n.4, p. 178 - 198. Edição Especial. Canoas, 2014.

SÁPIRAS, F. S.; DALLA VECCHIA, R.; MALTEMPI, M. V. Utilização do Scratch em sala de aula. Revista Educação Matemática Pesquisa, v. 17, p. 973 - 988, 2015.

SINCLAIR, N.; JACKIW, N. Modeling Practicies with The Geometer's Sketchpad. In: LESH, Richard; GALBRAITH, Peter; HAINES, Christopher R.; HURFORD, Andrew. (Org.). Modeling Students' Mathematical Modeling Competences. New York: U.S.A., Springer, 2010.

\section{Biografia Resumida}

Rose Grochot Gayeski: Licenciada em Matemática pela Universidade de Passo Fundo (2007). Especialista em Matemática, Mídias Digitais e Didática: tripé para a formação do professor de Matemática (2010). Mestra em Ensino de Matemática pela Universidade Federal do Rio Grande do Sul (2019). Atualmente sou professora das disciplinas de Cálculo e Estatística e diretora de ensino da Faculdade CESURG de Marau. Também, sou professora da disciplina de matemática na Escola de Ensino Médio Rainha D' Paz. Desenvolvo pesquisas na área de educação matemática atuando principalmente nos seguintes temas: Modelagem Matemática, Tecnologia, Literacia Digital, Big Data, Narrativas, Interdisciplinaridade e Estatística.

Lattes: http://lattes.cnpq.br/9405361404496368

Contato: seduc.proferose@gmail.com

Rodrigo Dalla Vecchia: Graduação em Matemática Licenciatura Plena pela Universidade Luterana do Brasil (2002). Mestrado em Matemática pela Universidade Federal do Rio Grande do Sul (2005). Doutorado em Educação Matemática pela Universidade Estadual Paulista Julio de Mesquita Filho (UNESP) de Rio Claro. Atualmente é professor da Universidade Federal do Rio Grande ISSN 2526-2882 
do Sul (UFRGS), atuando no curso de Licenciatura em Matemática. Desenvolve pesquisas na área de Educação Matemática, atuando principalmente nos seguintes temas: Modelagem Matemática, Tecnologias da Informação e Comunicação, Big Data Machine Learning e Construção de Jogos Eletrônicos.

Lattes: http://lattes.cnpq.br/3543447716539874

Contato: rodrigovecchia@gmail.com

Marcus Vinicius Maltempi: Graduado em Ciência da Computação pela Unesp, mestre em Ciências da Computação e Matemática Computacional pela USP, doutor em Engenharia Elétrica e de Computação pela Unicamp, tendo realizado parte do doutoramento na Universidade de Toronto (Canadá). Fez pósdoutorado na Universidade de Londres (Inglaterra), é Livredocente em Educação Matemática pela Unesp. Atualmente é Professor Associado III da Unesp de Rio Claro, ViceCoordenador Executivo do Instituto de Educação e Pesquisa em Práticas Pedagógicas (IEP-Unesp), Coordenador Geral da Unesp do Sistema Universidade Aberta do Brasil (UAB-Capes) e membro titular da Câmara de $\mathrm{EaD} / \mathrm{UAB}$ da Abruem. Foi ViceChefe de Departamento, Coordenador do Curso de Ciências da Computação, Coordenador do Programa de Pós-graduação em Educação Matemática e Coordenador do Núcleo de Educação a Distância (NEaD-Unesp). Editor-chefe das Revistas Bolema (Boletim de Educação Matemática) e da InFor - Inovação e Formação. Possui projetos em andamento com financiamento de agências de fomento (Capes e Fapesp). Tem experiência na área de Informática na Educação, mais especificamente em Educação Matemática e Educação a Distância.

Lattes: http://lattes.cnpq.br/5990590753771856

Contato: marcus.maltempi@unesp.br 